\title{
On the convergence of multipoint Padé-type approximants and quadrature formulas associated with the unit circle*
}

\author{
A. Bultheel ${ }^{\dagger}$ P. González-Vera ${ }^{\dagger}$ E. Hendriksen, O. Njåstad ${ }^{\S}$
}

\begin{abstract}
We study the convergence of rational interpolants with prescribed poles on the unit circle to the Herglotz-Riesz transform of a complex measure supported on $[-\pi, \pi]$. As a consequence, quadrature formulas arise which integrate exactly certain rational functions. Estimates of the rate of convergence of these quadrature formulas are also included.
\end{abstract}

Keywords: multipoint Padé-type approximation, orthogonal rational functions, quadrature formula.

AMS Classification: 41A21, 30E05, 41A55.

\section{Introduction}

Let $\mu$ be a finite complex Borel measure supported on $[-\pi, \pi]$, thus with $S_{\mu}=\operatorname{supp}(\mu) \subset$ $[-\pi, \pi]$ and $\int_{-\pi}^{\pi} d|\mu|=K<\infty$, where $|\mu|$ is the total variation measure. The Herglotz-Riesz transform of $\mu$ is

$$
F_{\mu}(z)=\int_{-\pi}^{\pi} \frac{e^{i \theta}+z}{e^{i \theta}-z} d \mu(\theta) .
$$

We shall approximate (1.1) by rational functions with prescribed poles (multipoint Padétype approximants $=$ MPTA). This gives rise to quadrature formulas of the form (2.1) below to estimate integrals on the unit circle of the form

$$
I_{\mu}(f)=\int_{-\pi}^{\pi} f\left(e^{i \theta}\right) d \mu(\theta) .
$$

For these formulas, it will be shown that they are exact for certain rational functions with given poles. Motivated by a paper of Nuttal and Wherry [17] (see also [12]), we treated a similar problem in [4] where the quadrature formulas integrated exactly Laurent polynomials and the approximants were restricted to two-point Padé approximants for (1.1). In Section

\footnotetext{
${ }^{*}$ This research was performed as part of the European project ROLLS under contract CHRX-CT93-0416.

${ }^{\dagger}$ Department of Computer Science, K.U.Leuven, Belgium

${ }^{\ddagger}$ Department Análisis Math., Univ. La Laguna, Tenerife, Spain

$\S$ Department of Math., Univ. of Amsterdam, The Netherlands

"Department of Math. Sciences, Univ. of Trondheim - NTH, Norway
} 
2, we give the connection between quadrature formulas and MPTAs to (1.1). Estimations of the rate of convergence of MPTAs to $F_{\mu}(z)$ are provided in Section 3. This will also result in an estimate of the rate of convergence of the quadrature formulas when the integrand $f$ is a holomorphic function in a neigborhood of $S_{\mu}$. In Section 4, we study the case when $f$ is only continuous with a modulus of continuity that satisfies certain conditions.

\section{Preliminary results}

We use the following notation for the unit circle, its inside and its outside: $\mathbb{T}=\{z \in \mathbb{C}$ : $|z|=1\}, \mathbb{D}=\{z \in \mathbb{C}:|z|<1\}$, and $\mathbb{E}=\{z \in \mathbb{C}:|z|>1\}$. $\mathbb{C}$ is the set of complex numbers and $\hat{\mathbb{C}}=\mathbb{C} \cup\{\infty\}$. The space of polynomials of degree at most $n$ is denoted as $\Pi_{n}$ and $\Pi$ is the space of all polynomials. For every pair of integers $(p, q), p \leq q$, we denote by $\Lambda_{p, q}$ the linear space of all Laurent polynomials (L-polynomials) of the form $L(z)=\sum_{j=p}^{q} c_{j} z^{j}$, $c_{j} \in \mathbb{C}$. $\Lambda$ is the space of all L-polynomials. Our quadrature formulas, approximating (1.2) will be of the form

$$
I_{n}(f)=\sum_{j=1}^{n} A_{j, n} f\left(x_{j, n}\right), \quad x_{j, n} \in \mathbb{T}, \quad x_{i, n} \neq x_{j, n}, \quad \text { for } i \neq j .
$$

For its construction we shall not use the L-polynomials as in [4] but we shall use rational functions with poles not on $\mathbb{T}$. For quadrature formulas based on rational functions with prescribed poles outside a finite interval of the real line, see e.g. $[14,22,8]$. Let $\alpha=\left\{\alpha_{n}\right.$ : $n=1,2, \ldots\}$ be an arbitrary sequence in $\mathbb{D}$. We introduce the Blaschke factors $\zeta_{k}(z)$ as

$$
\zeta_{k}(z)=\frac{\bar{\alpha}_{k}}{\left|\alpha_{k}\right|} \frac{\alpha_{k}-z}{1-\bar{\alpha}_{k} z}, \quad k=1,2, \ldots
$$

and the Blaschke products $B_{0}=1$, and $B_{k}=\zeta_{1} \ldots \zeta_{k}$. These generate the spaces $\mathcal{L}_{n}=$ $\operatorname{span}\left\{B_{0}, \ldots, B_{n}\right\}$ and $\mathcal{L}=\cup_{0}^{\infty} \mathcal{L}_{n}$. Introducing $\pi_{0}=1$ and $\pi_{n}(z)=\Pi_{k=1}^{n}\left(1-\bar{\alpha}_{k} z\right)$, for $n>0$, we can represent $R \in \mathcal{L}_{n}$ as $R=q / \pi_{n}$, with $q \in \Pi_{n}$. The notation $f_{*}(z)=\overline{f(1 / \bar{z})}$ allows us to introduce the spaces $\mathcal{L}_{n *}=\operatorname{span}\left\{1, B_{1 *}, \ldots, B_{n *}\right\}$ and $\mathcal{L}_{*}=\cup_{1}^{\infty} \mathcal{L}_{n *}$. If $R \in \mathcal{L}_{n *}$, then $R=q / \omega_{n}$ with $q \in \Pi_{n}$ and where $\omega_{0}=1$ and $\omega_{n}(z)=\Pi_{j=1}^{n}\left(z-\alpha_{j}\right)$ for $n>0$. Furthermore, let $\mathcal{R}_{p, q}=\mathcal{L}_{p *}+\mathcal{L}_{q}=\left\{P /\left(\pi_{q} \omega_{p}\right): P \in \Pi_{p+q}\right\}$ and $\mathcal{R}=\mathcal{L}+\mathcal{L}_{*}$. Note that $\mathcal{R}_{p, q}=\operatorname{span}\left\{1 / B_{p}, \ldots, 1 / B_{1}, 1, B_{1}, \ldots, B_{q}\right\}$ and $\mathcal{R}_{0, n}=\mathcal{L}_{n}$. When all $\alpha_{k}=0$, then $\mathcal{R}_{p, q}=\Lambda_{-p, q}$ and $\mathcal{L}_{n}=\Pi_{n}$. The spaces $\mathcal{R}_{p, q}$ will play the same role in this paper as the L-polynomials in [4]. The following theorem is given in [1, Addendum A.2, p. 244] when all the $\alpha_{i}$ are different. The adaptation of the proof for the case when two or more points are allowed to coincide is trivial.

Theorem 2.1 The space $\mathcal{R}$ is dense in the space $C(\mathbb{T})$ of continuous functions on $\mathbb{T}$ if and only if $\sum\left(1-\left|\alpha_{k}\right|\right)=\infty$.

Let $X=\left\{x_{j, n}: j=1, \ldots, n ; n \in \mathbb{N}\right\}$ be a triangular array of points contained in $\mathbb{T}$ with $x_{j, n} \neq x_{k, n}$ for $j \neq k$ and let $F_{n}$ be a rational function of type $(n, n)$ with poles at $\left\{x_{j, n}\right\}_{j=1}^{n}$ interpolating $F_{\mu}(z)$ at $0, \alpha_{1}, \ldots, \alpha_{p}$ and at $\infty, 1 / \bar{\alpha}_{1}, \ldots, 1 / \bar{\alpha}_{q}$ such that $p+q=n-1$, with $p$ and $q$ nonnegative integers. Setting $F_{n}=Q_{n} / P_{n}$ with $P_{n}(z)=\Pi_{j=1}^{n}\left(z-x_{j, n}\right)$, if $\operatorname{deg}\left(\pi_{q}\right)=q$ then the polynomial $Q_{n} \in \Pi_{n}$ is uniquely defined by requiring that the function

$$
\frac{F_{\mu}(z) P_{n}(z)-Q_{n}(z)}{z \omega_{p}(z) \pi_{q}(z)}
$$


is analytic in $\hat{\mathbb{C}}-\mathbb{T}$ (to see this, just count the number of interpolation conditions). If $\ell=q-\operatorname{deg}\left(\pi_{q}\right)>0$, then one needs $\ell$ extra interpolation conditions at $\infty$ given by $F_{\mu}(z)-$ $F_{n}(z)=O\left(z^{-\ell-1}\right), z \rightarrow \infty$. This $F_{n}$ is called an $(n, n)$ MPTA to $F_{\mu}$ relative to the pair $(\alpha, X)$; we shall denote it by $(n / n)_{\mu}(z)$. Let

$$
F_{n}(z)=(n / n)_{\mu}(z)=\frac{Q_{n}(z)}{P_{n}(z)}=\lambda_{n}+\sum_{j=1}^{n} A_{j, n} \frac{x_{j, n}+z}{x_{j, n}-z}
$$

Then from $F_{n}(0)=\mu_{0}$ where $\mu_{0}=F_{\mu}(0)=\int_{-\pi}^{\pi} d \mu(\theta)$ and $F_{n}(\infty)=F_{\mu}(\infty)=-\mu_{0}$, it follows that $\lambda_{n}=0$. It is also easily checked that

$$
A_{j, n}=-\frac{1}{2 x_{j, n}} \frac{Q_{n}\left(x_{j, n}\right)}{P_{n}^{\prime}\left(x_{j, n}\right)}, \quad j=1,2, \ldots, n .
$$

Note that this equality holds for any partial fraction expansion of a rational function of the form (2.4) with $\lambda_{n}=0$.

In this section it will be shown that such an MPTA to $F_{\mu}$ leads to a quadrature formula which is exact in $\mathcal{R}_{p, q}$ and conversely that any quadrature formula exact in $\mathcal{R}_{p, q}$ can be obtained in this way.

Let the points of the sequence $\alpha$ be contained in a compact subset of $\mathbb{D}$ (in what follows, we paraphrase this by saying that $\alpha$ is compactly included in $\mathbb{D}$ ) and let $G$ be a region (open and connected $)$ in $\mathbb{C}$ such that $\mathbb{T} \subset G, 0 \notin G$, and $G \cap(\alpha \cup \hat{\alpha})=\emptyset$, where $\hat{\alpha}=\left\{1 / \bar{\alpha}_{k}\right\}_{1}^{\infty}$. Suppose that $\Gamma$, the boundary of $G$, is a finite union of Jordan curves, and suppose $f$ is a function analytic in $G$. From Cauchy's theorem, it then follows that

$$
f\left(z_{0}\right)=\frac{1}{2 \pi i} \int_{\Gamma} \frac{z_{0}+z}{z_{0}-z}\left(-\frac{f(z)}{2 z}\right) d z
$$

whenever $z_{0}$ is in $G$. From (2.6) and Fubini's theorem it follows that

$$
I_{\mu}(f)=\int_{-\pi}^{\pi} f\left(e^{i \theta}\right) d \mu(\theta)=\frac{1}{2 \pi i} \int_{\Gamma} F_{\mu}(z)\left(-\frac{f(z)}{2 z}\right) d z .
$$

Now consider an arbitrary function $R \in \mathcal{R}_{p, q}$ with $p+q=n-1$, then by taking into account that $F_{\mu}-(n / n)_{\mu}$ is analytic in $\hat{\mathbb{C}}-\mathbb{T}$, it follows from Cauchy's theorem that

$$
\int_{\Gamma}\left(F_{\mu}(z)-\frac{Q_{n}(z)}{P_{n}(z)}\right)\left(-\frac{R(z)}{2 z}\right) d z=0 .
$$

Thus, by (2.5)-(2.8)

$$
I_{\mu}(R)=\frac{1}{2 \pi i} \int_{\Gamma} F_{\mu}(z)\left(-\frac{R(z)}{2 z}\right) d z=\frac{1}{2 \pi i} \int_{\Gamma} \frac{Q_{n}(z)}{P_{n}(z)}\left(-\frac{R(z)}{2 z}\right) d z=\sum_{j=1}^{n} A_{j, n} R\left(x_{j, n}\right)=I_{n}(R) .
$$

Thus we obtained a quadrature formula (2.1) valid in $\mathcal{R}_{p, q}, p+q=n-1$. Furthermore, for any function $f$ analytic in $G$

$$
\begin{aligned}
I_{n}(f) & =\sum_{j=1}^{n} A_{j, n} f\left(x_{j, n}\right)=\sum_{j=1}^{n} A_{j, n}\left[\frac{1}{2 \pi i} \int_{\Gamma} \frac{x_{j, n}+z}{x_{j, n}-z}\left(-\frac{f(z)}{2 z}\right) d z\right] \\
& =\frac{1}{2 \pi i} \int_{\Gamma}\left[\sum_{j=1}^{n} A_{j, n} \frac{x_{j, n}+z}{x_{j, n}-z}\right]\left[-\frac{f(z)}{2 z}\right] d z=\frac{1}{2 \pi i} \int_{\Gamma}(n / n)_{\mu}(z)\left[-\frac{f(z)}{2 z}\right] d z .
\end{aligned}
$$


Remark 1. Starting from the table $X$, a formula (2.1) satisfying (2.9) for any $R \in \mathcal{R}_{p, q}$, $p+q=n-1$, can be constructed for any function $f$, defined on $\mathbb{T}$. However, representation (2.10) is only valid for an analytic function.

Remark 2. When all the $\alpha_{k}=0$, then $\mathcal{R}_{p, q}=\Lambda_{-p, q}$ and formula (2.9) coincides with those studied in [4] and [13].

For the error of (2.9), we immediately obtain

Lemma 2.2 Let $f$ be analytic in $G$, a neighborhood of $\mathbb{T}$ such that $G \cap(\alpha \cup \hat{\alpha})=\emptyset$ and $0 \notin G$. Then, for each $n$ and any triangular table $X \subset \mathbb{T}$ such that $x_{j, n} \neq x_{k, n}$ for $j \neq k$, we have

$$
E_{n}(f)=I_{\mu}(f)-I_{n}(f)=\frac{1}{2 \pi i} \int_{\Gamma}\left(F_{\mu}(z)-(n / n)_{\mu}(z)\right)\left(-\frac{f(z)}{2 z}\right) d z
$$

where $\Gamma$ is the boundary of $G$.

It trivially follows from (2.11) that

$$
\left|E_{n}(f)\right| \leq \frac{1}{4 \pi} \max _{x \in G} \frac{|f(x)|}{|x|} \int_{\Gamma}\left|F_{\mu}(z)-(n / n)_{\mu}(z)\right||d z| .
$$

So, we can say that a sequence $\left\{(n / n)_{\mu}\right\}$ of MPTAs that converges to $F_{\mu}$ uniformly on compact subsets of $\hat{\mathbb{C}}-\mathbb{T}$, can provide "suitable" quadrature formulas. The weights of the quadrature have the following integral representation

Theorem 2.3 Let $p$ and $q$ be nonnegative integers such that $p+q=n-1 \geq 0$. Let $I_{n}(f)=\sum_{1}^{n} A_{j, n} f\left(x_{j, n}\right)$ be a quadrature with distinct nodes $x_{j, n}$ on $\mathbb{T}$ that is exact in $\mathcal{R}_{p, q}$. Then

$$
A_{j, n}=\frac{\omega_{p}\left(x_{j, n}\right) \pi_{q}\left(x_{j, n}\right)}{P_{n}^{\prime}\left(x_{j, n}\right)} I_{\mu}\left(\frac{P_{n}(x)}{\omega_{p}(x) \pi_{q}(x)\left(x-x_{j, n}\right)}\right), \quad x=e^{i \theta}
$$

where $P_{n}(z)=\prod_{j=1}^{n}\left(z-x_{j, n}\right)$.

Proof. Let us consider the function (with variable $x$ and $z$ a parameter)

$$
R_{n}(x)=1+\frac{2 z}{x-z}\left(1-\frac{\omega_{p}(z) \pi_{q}(z) P_{n}(x)}{\omega_{p}(x) \pi_{q}(x) P_{n}(z)}\right)=\frac{x+z}{x-z}-\frac{2 z}{x-z} \frac{\omega_{p}(z) \pi_{q}(z) P_{n}(x)}{\omega_{p}(x) \pi_{q}(x) P_{n}(z)}
$$

Clearly, this function belongs to $\mathcal{R}_{p, q}$. Since the quadrature formula is assumed to be exact in $\mathcal{R}_{p, q}$ we obtain that $I_{\mu}\left(R_{n}\right)=I_{n}\left(R_{n}\right)$. Since $P\left(x_{j, n}\right)=0$, it follows that

$$
I_{\mu}\left(R_{n}\right)=\sum_{j=1}^{n} A_{j, n} \frac{x_{j, n}+z}{x_{j, n}-z}=\frac{Q_{n}(z)}{P_{n}(z)}
$$

where $Q_{n} \in \Pi_{n}$ is defined as the numerator polynomial of the rational function above. Thus one has

$$
Q_{n}(z)=P_{n}(z) I_{\mu}\left(R_{n}\right)=I_{\mu}\left(P_{n}(z)+\frac{2 z}{x-z}\left[P_{n}(z)-\frac{\omega_{p}(z) \pi_{q}(z) P_{n}(x)}{\omega_{p}(x) \pi_{q}(x)}\right]\right) .
$$

By using (2.5) and expression (2.16), for $Q_{n}$ we obtain (2.13). 
Remark 3. It can be easily verified that the Newton interpolation formula plus error term for the function $(t+z) /(t-z)$ is given by (see also [3, p. 41])

$$
\frac{t+z}{t-z}=1+2 \sum_{k=1}^{n} \frac{z \omega_{k-1}(z)}{\omega_{k}(t)}+2 \frac{z \omega_{n}(z)}{(t-z) \omega_{n}(t)}
$$

for $t \in \mathbb{T}$ and $z \neq t$. Similarly (e.g., by applying the substar conjugate) we get

$$
\frac{t+z}{t-z}=-1-2 \sum_{k=1}^{n} \frac{t^{k} \pi_{k-1}(z)}{z^{k} \pi_{k}(t)}+2 \frac{t^{n+1} \pi_{n}(z)}{z^{n}(t-z) \pi_{n}(t)} .
$$

Now a converse to (2.9) can be given. Indeed, let the quadrature (2.1) be exact in $\mathcal{R}_{p, q}$, $p+q=n-1$ and let $F_{n}=Q_{n} / P_{n}$ the rational function as defined in Theorem 2.3. Assume that $n>1$ (the case $n=1$ is trivial) and take $p>0$. Then, making use of (2.17) one can write

$$
\begin{aligned}
F_{n}(z) & =\sum_{j=1}^{n} A_{j, n} \frac{x_{j, n}+z}{x_{j, n}-z} \\
& =\mu_{0}+2 \sum_{k=1}^{p} z \omega_{k-1}(z) I_{\mu}\left(1 / \omega_{k}(t)\right)+2 z \omega_{p}(z) \sum_{j=1}^{p} \frac{A_{j, n}}{\left(x_{j, n}-z\right) \omega_{p}\left(x_{j, n}\right)}
\end{aligned}
$$

and

$$
F_{\mu}(z)=\mu_{0}+2 \sum_{k=1}^{p} z \omega_{k-1}(z) I_{\mu}\left(1 / \omega_{k}(t)\right)+2 z \omega_{p}(z) I_{\mu}\left(\frac{1}{(t-z) \omega_{p}(t)}\right) .
$$

Similarly, using (2.18) and supposing $q>0$, we can get

$$
F_{n}(z)=-\mu_{0}-2 \sum_{k=1}^{q} \frac{\pi_{k-1}(z)}{z^{k}} I_{\mu}\left(\frac{t^{k}}{\pi_{k}(t)}\right)+2 \frac{\pi_{q}(z)}{z^{q}} \sum_{j=1}^{q} \frac{A_{j, n} x_{j, n}^{q+1}}{\left(x_{j, n}-z\right) \pi_{q}\left(x_{j, n}\right)}
$$

and

$$
F_{\mu}(z)=-\mu_{0}-2 \sum_{k=1}^{q} \frac{\pi_{k-1}(t)}{z^{k}} I_{\mu}\left(\frac{t^{k}}{\pi_{k}\left(x_{j, n}\right)}\right)+2 \frac{\pi_{q}(z)}{z^{q}} I_{\mu}\left(\frac{t^{q+1}}{(t-z) \pi_{q}(t)}\right) .
$$

Thus, from (2.19-2.20) and (2.21-2.22), it can be easily deduced that $F_{n}(z)$ is an MPTA to $F_{\mu}$.

Remark 4. Let $R_{p, q} \in \mathcal{R}_{p, q}, p+q=n-1$ be an interpolant for some function $f$ defined on $\mathbb{T}$ with interpolation points $\left\{x_{j, n}\right\}$ with $x_{j, n} \neq x_{k, n}$ for $j \neq k$ and $X \cap(\alpha \cup \hat{\alpha})=\emptyset$. Thus $R_{p, q}\left(x_{j, n}\right)=f\left(x_{j, n}\right), j=1, \ldots, n$. Integration of $R_{p, q}\left(e^{i \theta}\right)$ with respect to $d \mu(\theta)$ produces a quadrature formula $I_{n}(f)$ exact in $\mathcal{R}_{p, q}$. This fact enables us to obtain (2.13) in a different way. We have indeed (for further details, see $[2, \S 2]$ ):

$$
R_{p, q}(z)=\sum_{j=1}^{n} L_{j, n}^{p}(z) f\left(x_{j, n}\right)
$$

where

$$
L_{j, n}^{p}(z)=\frac{1-\bar{\alpha}_{q+1} z}{1-\bar{\alpha}_{q+1} x_{j, n}} \frac{\Omega_{n}(z)}{\left(z-x_{j, n}\right) \Omega_{n}^{\prime}\left(x_{j, n}\right)} \in \mathcal{R}_{p, q}
$$


with

$$
\Omega_{n}(z)=\frac{P_{n}(z)}{\omega_{p}(z) \pi_{q+1}(z)} \in \mathcal{R}_{p, q+1}
$$

Since

$$
A_{j, n}=I_{\mu}\left(L_{j, n}^{p}\right), \quad \text { and } \quad \Omega_{n}^{\prime}\left(x_{j, n}\right)=\frac{P_{n}^{\prime}\left(x_{j, n}\right)}{\omega_{p}\left(x_{j, n}\right) \pi_{q+1}\left(x_{j, n}\right)}
$$

and $\pi_{q+1}(z)=\pi_{q}(z)\left(1-\bar{\alpha}_{q+1} z\right)$, formula $(2.13)$ easily follows.

To conclude this section, we give an integral representation for the error of the MPTA.

Lemma 2.4 Let $\alpha$ and $X$ be as above. Then, setting $x=e^{i \theta}$, we have

$$
F_{\mu}(z)-(n / n)_{\mu}(z)=F_{\mu}(z)-\frac{Q_{n}(z)}{P_{n}(z)}=\frac{2 z \omega_{p}(z) \pi_{q}(z)}{P_{n}(z)} \int_{-\pi}^{\pi} \frac{P_{n}(x) d \mu(\theta)}{\omega_{p}(x) \pi_{q}(x)(x-z)} .
$$

Proof. This follows from (1.1), Remark 3 and (2.16).

\section{Convergence for analytic functions}

In this section we shall give results about the rate of convergence for the MPTAs $(n / n)_{\mu}$ to $F_{\mu}$. These will immediately imply an estimate for the rate of convergence of the quadrature formula $I_{n}(f)$ to the integral $I_{\mu}(f)$ when $f$ is a function analytic in a neighborhood of $\mathbb{T}$.

As before, $\alpha$ will denote a sequence of points compactly included in $\mathbb{D}$ and $X$ a triangular array of points on $\mathbb{T}$. We want to make appropriate selections of $\alpha$ and $X$ to obtain good estimates of the MPTA $(n / n)_{\mu}$ and deduce bounds for the rate of convergence of the corresponding quadrature formulas for some function $f$ holomorphic in a neighborhood of T.

With a polynomial $Q \in \Pi_{n}$, we associate the normalized counting measure $\nu_{n}(Q)=$ $\frac{1}{n} \sum_{Q(\xi)=0} \delta_{\xi}$ where $\delta_{\xi}$ is the Dirac measure supported at $\xi$. It assigns a point mass at the zeros of the polynomial $Q$, counting multiplicity. For $Q=\omega_{n}$, we denote this measure as $\nu_{n}^{\alpha}$. The logarithmic potential of a measure $\nu$ is given by $V_{\nu}(z)=-\int \log |z-\xi| d \nu(\xi)$. It is obvious that we have $\left|\omega_{n}(z)\right|^{1 / n}=\exp \left\{-V_{\nu_{n}^{\alpha}}(z)\right\}$. Assume that there exists a measure $\nu^{\alpha}$ such that $\nu_{n}^{\alpha}$ converges to $\nu^{\alpha}$ in the weak star topology of the space of measures. We shall denote this as

$$
\nu_{n}^{\alpha} \stackrel{*}{\longrightarrow} \nu^{\alpha} .
$$

This convergence implies [23, Lemma 1, p. 436]

$$
\lim _{n \rightarrow \infty}\left|\omega_{n}(z)\right|^{1 / n}=\exp \left\{-V_{\nu^{\alpha}}(z)\right\}, \quad z \in \mathbb{C}-\operatorname{supp}\left(\nu^{\alpha}\right)
$$

and

$$
\limsup _{n \rightarrow \infty}\left|\omega_{n}(z)\right|^{1 / n} \leq \exp \left\{-V_{\nu^{\alpha}}(z)\right\}, \quad z \in \mathbb{C} .
$$

Convergence is uniform on each compact subset of the indicated regions. Set $\bar{\omega}_{n}(z)=$ $\Pi_{j=1}^{n}\left(z-\bar{\alpha}_{j}\right)$ (this equals $z^{n} \pi_{n}(1 / z)$ if $\left.z \neq 0\right)$. Let $\nu^{\bar{\alpha}}$ be the measure associated with the point set $\bar{\alpha}$, just as $\nu^{\alpha}$ was associated with $\alpha$. Then for any $z$ such that $1 / z \in(\mathbb{C}-\{0\})-\operatorname{supp}\left(\nu^{\bar{\alpha}}\right)$ we have

$$
\lim _{n \rightarrow \infty}\left|\pi_{n}(z)\right|^{1 / n}=|z| \lim _{n \rightarrow \infty}\left|\bar{\omega}_{n}(1 / z)\right|^{1 / n}=|z| \exp \left\{-V_{\nu^{\bar{\alpha}}}(1 / z)\right\}
$$


Furthermore, $\lim _{n \rightarrow \infty}\left|\pi_{n}(0)\right|^{1 / n}=1$ and

$$
\limsup _{n \rightarrow \infty}\left|\pi_{n}(z)\right|^{1 / n} \leq|z| \exp \left\{-V_{\nu^{\bar{\alpha}}}(1 / z)\right\}, \quad z \in \mathbb{C}-\{0\} .
$$

As before, $p$ and $q$ are nonnegative integers such that $p+q=n-1 \geq 0$. Since we shall now let $n \rightarrow \infty$, these $p$ and $q$ which are functions of $n$, will be written explicitly as $p(n)$ and $q(n)$. Now suppose that $\lim _{n \rightarrow \infty}(p(n) / n)=r$ and $\lim _{n \rightarrow \infty}(q(n) / n)=s$. Since $p(n)+q(n)=n-1$, we should have $r+s=1$. Then, it follows from the above results that we have for $z \in(\mathbb{C}-\{0\})-\operatorname{supp}\left(\nu^{\alpha}\right)$ and $1 / z \notin \operatorname{supp}\left(\nu^{\bar{\alpha}}\right)$

$$
\lim _{n \rightarrow \infty}\left|\omega_{p}(z) \pi_{q}(z)\right|^{1 / n}=\exp \left\{-\left(r V_{\nu^{\alpha}}(z)+s V_{\nu^{\alpha}}(1 / z)\right)\right\}|z|^{s}
$$

and for $z \neq 0$

$$
\limsup _{n \rightarrow \infty}\left|\omega_{p}(z) \pi_{q}(z)\right|^{1 / n} \leq \exp \left\{-\left(r V_{\nu^{\alpha}}(z)+s V_{\nu^{\bar{\alpha}}}(1 / z)\right)\right\}|z|^{s} .
$$

We shall now concentrate on the case $\lim _{n \rightarrow \infty} \alpha_{n}=a$. Without loss of generality, we may assume $a=0$. Hence $\nu^{\alpha}=\delta_{0}=\nu^{\bar{\alpha}}$ and $\operatorname{supp}\left(\nu^{\alpha}\right)=\{0\}$. Since $V_{\delta_{0}}(z)=-\log |z|$, we have by $(3.4)$

$$
\lim _{n \rightarrow \infty}\left|\omega_{p}(z) \pi_{q}(z)\right|^{1 / n}=|z|^{r}, \quad z \in \mathbb{C}-\{0\},
$$

where convergence is uniform on compact subsets of $\mathbb{C}-\{0\}$. From (2.24) we deduce

$$
\left|F_{\mu}(z)-(n / n)_{\mu}(z)\right| \leq \frac{2|z|\left|\omega_{p}(z) \pi_{q}(z)\right|}{\left|P_{n}(z)\right|} \frac{\max _{x \in \mathbb{T}}\left|P_{n}(x)\right|}{\min _{x \in \mathbb{T}}\left|\omega_{p}(x) \pi_{q}(x)\right|} \int_{-\pi}^{\pi} \frac{d|\mu|(\theta)}{\mid z-e^{i \theta \mid}},
$$

and, consequently, taking limits for $n \rightarrow \infty$, we have

$$
\begin{aligned}
\lim \sup \left|F_{\mu}(z)-(n / n)_{\mu}(z)\right|^{1 / n} & \leq \frac{\lim \sup \left|\omega_{p}(z) \pi_{q}(z)\right|^{1 / n}}{\lim \inf \left|P_{n}(z)\right|^{1 / n}} \frac{\lim \sup \left\{\max _{x \in \mathbb{T}}\left|P_{n}(x)\right|\right\}^{1 / n}}{\lim \inf \left\{\min _{x \in \mathbb{T}}\left|\omega_{p}(x) \pi_{q}(x)\right|\right\}^{1 / n}} \\
& \leq \frac{|z|^{r}}{\lim \inf \left|P_{n}(z)\right|^{1 / n}} \lim \sup \left\{\max _{x \in \mathbb{T}}\left|P_{n}(x)\right|\right\}^{1 / n}
\end{aligned}
$$

by (3.6). This shows that we have to investigate the $n$th root asymptotic behavior of the polynomial $P_{n}(z)$, so that this upper bound is less than 1 on any compact subset of $\hat{\mathbb{C}}-\mathbb{T}$, and thus that geometric convergence results. An appropriate selection for the nodes $X$ will correspond to the zeros of the para-orthogonal polynomials. We introduce these first.

Let $\psi$ be a finite positive Borel measure on $\mathbb{T}$ and let $\left\{\rho_{n}\right\}$ be the corresponding sequence of monic Szegö polynomials. The normalized polynomials are $\phi_{n}(z)=\kappa_{n} \rho_{n}(z), \kappa_{n}>0$. Thus $\left\langle\phi_{k}, \phi_{l}\right\rangle_{\psi}=\delta_{k, l}$, where $\langle f, g\rangle_{\psi}=\int_{-\pi}^{\pi} f\left(e^{i \theta}\right) \overline{g\left(e^{i \theta}\right)} d \psi(\theta)$. We introduce the notation $\phi_{n}^{*}(z)=z^{n} \phi_{n *}(z)$. If $\tau \in \mathbb{T}$, then $\chi_{n}(z, \tau)=\phi_{n}(z)+\tau \phi_{n}^{*}(z)$ is called the $n$th para-orthogonal polynomial $[15, \S 6]$. It is orthogonal to $\operatorname{span}\left\{z, \ldots, z^{n-1}\right\}$. For the convergence result to be given in Theorem 3.4, we need the $n$th root asymptotics for the para-orthogonal polynomials $\chi_{n}$. Such asymptotics can be obtained under rather week conditions for the measure $\psi$ (see $[20$, Ch. 3]). However, since the measure $\psi$ only plays an auxiliary role in the present paper (see Theorem 3.4), it is not essential that we have the weakest possible condition on the measure $\psi$. It is sufficient to know that such a measure exists. So, we shall follow a paved road and assume that $\psi^{\prime}>0$ a.e., which is a sufficient condition to prove the ratio asymptotics for the $\chi_{n}$, which of course implies the $n$ th-root asymptotics we need. This will be done in Theorem 3.3. For its proof we need the following lemma's: 
Lemma 3.1 With the above notation and on condition that $\psi^{\prime}(\theta)>0$ almost everywhere on $[-\pi, \pi]$, we have
(i) $\quad \lim _{n \rightarrow \infty} \frac{\phi_{n+1}(z)}{\phi_{n}(z)}=z \quad$ uniformly on $\mathbb{T} \cup \mathbb{E}$
(ii) $\lim _{n \rightarrow \infty} \frac{\phi_{n}^{*}(z)}{\phi_{n}(z)}=0$ uniformly on compact subsets of $\mathbb{E}$
(iii) $\quad \lim _{n \rightarrow \infty} \frac{\phi_{n+1}^{*}(z)}{\phi_{n}^{*}(z)}=1 \quad$ uniformly on $\mathbb{T} \cup \mathbb{D}$
(iv) $\lim _{n \rightarrow \infty} \frac{\phi_{n}(z)}{\phi_{n}^{*}(z)}=0$ uniformly on compact subsets of $\mathbb{D}$
(v) $\quad \lim _{n \rightarrow \infty} \rho_{n}(0)=0$ and $\lim _{n \rightarrow \infty} \kappa_{n+1} / \kappa_{n}=1$.

Statements (i) and (iii) and the statements in (v) are equivalent (see [16, Lemma 2.4] and the comments following it). Also (ii) and (iv) are equivalent and follow from (v) [18, Lemma 6]. That $\psi^{\prime}>0$ a.e. implies (i) is given in [18, Thm. 1, $4^{\circ}$, p. 207] (see also [16, Lemma 2.4]); that it implies (v) is given in [19, Theorem on p. 206].

Lemma 3.2 (see [24, § 7.4]) Let $K$ be a compact subset of $\mathbb{C}$ and $\left\{P_{n}(z)\right\}$ a sequence of monic polynomials so that for each $n$, the zeros of $P_{n}(z)$ lie in $K$. Let $\left\|P_{n}\right\|_{K}=$ $\max _{x \in K}\left|P_{n}(z)\right|$ denote the maximum norm in $K$, let $g_{k}(z ; \infty)$ be the Green's function for $K$ with pole at $\infty$, and let $\operatorname{Cap}(K)$ be the logarithmic capacity of $K$. Then we have $\lim _{n \rightarrow \infty}\left|P_{n}(z)\right|^{1 / n}=$ $\exp \left\{g_{K}(z ; \infty)\right\} \operatorname{Cap}(K)$ uniformly on any compact subset of $\mathbb{C}-K$ if and only if $\lim _{n \rightarrow \infty}\left\|P_{n}\right\|_{K}^{1 / n}=$ $\operatorname{Cap}(K)$.

Now we can formulate

Theorem 3.3 Let $\left\{\tau_{n}\right\}_{1}^{\infty}$ be a sequence of complex numbers on $\mathbb{T}$. Set $\chi_{n}^{\psi}\left(z, \tau_{n}\right)=\chi_{n}(z)=$ $\phi_{n}(z)+\tau_{n} \phi_{n}^{*}(z)$ the para-orthogonal polynomials with respect to $\psi$. Assume $\psi^{\prime}>0$ a.e. Then we have

(i) $\quad \lim _{n \rightarrow \infty}\left|\chi_{n}(z)\right|^{1 / n}=|z| \quad$ uniformly on compact subsets of $\mathbb{E}$

(ii) $\quad \lim _{n \rightarrow \infty}\left|\chi_{n}(z)\right|^{1 / n}=1 \quad$ uniformly on compact subsets of $\mathbb{D}$

(iii) $\quad \lim _{n \rightarrow \infty} M_{n}^{1 / n}=1 \quad$ where $M_{n}=\left\|\chi_{n}\right\|_{\mathbb{T}}$.

Proof. (i) Take $z \in \mathbb{E}$, then

$$
\frac{\chi_{n+1}(z)}{\chi_{n}(z)}=\frac{\phi_{n+1}(z)+\tau_{n+1} \phi_{n+1}^{*}(z)}{\phi_{n}(z)+\tau_{n} \phi_{n}^{*}(z)}=\frac{\phi_{n+1}(z)}{\phi_{n}(z)} \frac{1+\tau_{n+1} \phi_{n+1}^{*}(z) / \phi_{n+1}(z)}{1+\tau_{n} \phi_{n}^{*}(z) / \phi_{n}(z)}
$$

Thus by (i) and (ii) of Lemma 3.1, it follows that $\lim _{n \rightarrow \infty} \chi_{n+1}(z) / \chi_{n}(z)=z$, and therefore also $\lim _{n \rightarrow \infty}\left|\chi_{n+1}(z)\right|^{1 / n}=|z|$.

(ii) As in (i) we have

$$
\frac{\chi_{n+1}(z)}{\chi_{n}(z)}=\frac{\phi_{n+1}^{*}(z)}{\phi_{n}^{*}(z)} \frac{\tau_{n+1}+\phi_{n+1}(z) / \phi_{n+1}^{*}(z)}{\tau_{n}+\phi_{n}(z) / \phi_{n}^{*}(z)} .
$$


The second fraction goes to 1, i.e.,

$$
\lim _{n \rightarrow \infty}\left|\frac{\tau_{n+1}+\phi_{n+1}(z) / \phi_{n+1}^{*}(z)}{\tau_{n}+\phi_{n}(z) / \phi_{n}^{*}(z)}\right|=1
$$

because for sufficiently large $n$

$$
\frac{1-\left|\phi_{n+1}(z) / \phi_{n+1}^{*}(z)\right|}{1+\left|\phi_{n}(z) / \phi_{n}^{*}(z)\right|} \leq\left|\frac{\tau_{n+1}+\phi_{n+1}(z) / \phi_{n+1}^{*}(z)}{\tau_{n}+\phi_{n}(z) / \phi_{n}^{*}(z)}\right| \leq \frac{1+\left|\phi_{n+1}(z) / \phi_{n+1}^{*}(z)\right|}{1-\left|\phi_{n}(z) / \phi_{n}^{*}(z)\right|}
$$

so that ii) follows by iv) of Lemma 3.1 .

(iii) Let $\lambda_{n}=\kappa_{n}+\tau_{n} \overline{\phi_{n}(0)}=\kappa_{n}\left(1+\tau_{n} \overline{\rho_{n}(0)}\right)$ be the leading coefficient of $\chi_{n}(z)$. Thus

$$
\frac{\lambda_{n+1}}{\lambda_{n}}=\frac{\kappa_{n+1}}{\kappa_{n}} \frac{1+\tau_{n+1} \overline{\rho_{n+1}(0)}}{1+\tau_{n} \overline{\rho_{n}(0)}}
$$

and because $\lim _{n \rightarrow \infty} \rho_{n}(0)=0$ by $\mathrm{v}$ ) of Lemma 3.1, we get that

$$
\lim _{n \rightarrow \infty} \frac{\lambda_{n+1}}{\lambda_{n}}=\lim _{n \rightarrow \infty} \frac{\kappa_{n+1}}{\kappa_{n}}=1
$$

so that

$$
\lim _{n \rightarrow \infty}\left|\lambda_{n}\right|^{1 / n}=1 \text {. }
$$

The sequence $\left\{\chi_{n}(z) / \lambda_{n}\right\}$ of monic polynomials satisfies $\lim _{n \rightarrow \infty}\left|\chi_{n}(z) / \lambda_{n}\right|^{1 / n}=|z|$. Using Lemma 3.2 with $K=\mathbb{D} \cup \mathbb{T}$, we get

$$
1=\lim _{n \rightarrow \infty}\left\|\chi_{n} / \lambda_{n}\right\|_{\mathbb{T}}=\lim _{n \rightarrow \infty} \frac{1}{\left|\lambda_{n}\right|^{1 / n}}\left\|\chi_{n}\right\|_{\mathbb{T}}^{1 / n}=\lim _{n \rightarrow \infty}\left\|\chi_{n}\right\|_{\mathbb{T}}^{1 / n}
$$

and this concludes the proof.

We thus have

Theorem 3.4 Let $\alpha=\left\{\alpha_{n}\right\}$ be a sequence of points contained in $\mathbb{D}$ and $\lim _{n \rightarrow \infty} \alpha_{n}=0$. For each $n$, let $\left\{x_{j, n}\right\}$ be the zeros of the nth para-orthogonal polynomial $\chi_{n}\left(z ; \tau_{n}\right), \tau_{n} \in \mathbb{T}$, associated with some positive Borel measure $\psi$ on $\mathbb{T}$ as described above. Furthermore, let $F_{n}(z)=Q_{n}(z) / P_{n}(z)=(n / n)_{\mu}(z)$ be a MPTA for $F_{\mu}(z)$ with denominator $P_{n}(z)=\Pi_{1}^{n}(z-$ $\left.x_{j, n}\right)$. Assume $\psi^{\prime}>0$ a.e. Then we have

$$
\limsup _{n \rightarrow \infty}\left|F_{\mu}(z)-(n / n)_{\mu}(z)\right|^{1 / n} \leq \lambda(z)<1
$$

where $\lambda(z)=|z|^{r}$ if $z \in \mathbb{D}$ and $\lambda(z)=|z|^{r-1}$ if $z \in \mathbb{E}$. This limit holds uniformly on compact subsets of $\hat{\mathbb{C}}-\mathbb{T}$.

Proof. Make use of (3.7) and Theorem 3.3.

Corollary 3.5 Let $f$ be analytic in a neighborhood $G$ of $\mathbb{T}$ and $0 \notin G$. Assume that $\alpha$ and $X$ satisfy the conditions of Theorem 3.4. Let $I_{n}(f)$ denotes the n-point quadrature formula with nodes $\left\{x_{j, n}\right\}_{1}^{n}$ valid in $\mathcal{R}_{p(n), q(n)}$ with $p(n)+q(n)=n-1$ and suppose $\lim _{n \rightarrow \infty} p(n) / n=r$, $0<r<1$. Then,

$$
\limsup _{n \rightarrow \infty}\left|E_{n}(f)\right|^{1 / n}=\limsup _{n \rightarrow \infty}\left|I_{\mu}(f)-I_{n}(f)\right|^{1 / n} \leq \gamma<1
$$

where $\gamma=\max \left\{\gamma_{1}, \gamma_{2}\right\}$ with $\gamma_{1}=\max \left\{|z|^{r}: z \in \Gamma \cap \mathbb{D}\right\}, \gamma_{2}=\max \left\{|z|^{r-1}: z \in \Gamma \cap \mathbb{E}\right\}$ and $\Gamma$ is the boundary of $G$.

Proof. See (2.11) and Theorem 3.4. 


\section{Convergence for continuous functions}

In this section, we shall prove similar convergence results when $f$ is continuous on $\mathbb{T}$, but not necessarily analytic. For simplicity, we shall only consider complex measures of the form $d \mu(\theta)=w(\theta) d \theta$ where $w$ is a complex-valued measurable function on $[-\pi, \pi]$ such that

$$
\int_{-\pi}^{\pi}|w(\theta)| d \theta<\infty
$$

Let $h(\theta)$ be a weight function defined on $[-\pi, \pi]$, i.e., $h(\theta)>0$ a.e. and

$$
\int_{-\pi}^{\pi} h(\theta) d \theta<\infty
$$

Assume that

$$
\int_{-\pi}^{\pi} \frac{|w(\theta)|^{2}}{h(\theta)} d \theta=C_{1}<\infty
$$

Let us consider a sequence $\alpha=\left\{\alpha_{n}\right\}$ compactly included in $\mathbb{D}$. From the inner product associated with $h$, i.e.,

$$
\langle f, g\rangle=\int_{-\pi}^{\pi} f\left(e^{i \theta}\right) \overline{g\left(e^{i \theta}\right)} h(\theta) d \theta,
$$

we generate an orthonormal system of rational functions $\left\{\phi_{k}\right\}, \phi_{0}=1$ and $\phi_{k} \in \mathcal{L}_{k}-\mathcal{L}_{k-1}$ and $\phi_{k} \perp \mathcal{L}_{k-1}, k=1,2, \ldots$. We introduce the notation $\phi_{n}^{*}(z)=B_{n}(z) \phi_{n *}(z)$ with $B_{n}$ the finite Blaschke product with zeros $\alpha_{1}, \ldots, \alpha_{n}$. The $\phi_{k}$ are uniquely defined if we require that $\kappa_{n}=\phi_{n}^{*}\left(\alpha_{n}\right)>0$. Thus if $\phi_{n}(z)=\sum_{k=0}^{n} a_{k, n} B_{k}(z)$, then $a_{n, n}=\kappa_{n}$. In analogy with the polynomial case where all $\alpha_{k}=0$, we call $\kappa_{n}$ the leading coefficient of $\phi_{n}$ (with respect to the basis $B_{k}$ ). Also in analogy with the polynomial case we set for $\tau_{n} \in \mathbb{T}$

$$
f_{n}\left(z ; \tau_{n}\right)=\phi_{n}(z)+\tau_{n} \phi_{n}^{*}(z) \in \mathcal{L}_{n} .
$$

Functions defined by (4.5) are called para-orthogonal rational functions. We have from $[2$, Theorem 4]

Theorem 4.1 For $\tau_{n} \in \mathbb{T}$, the para-orthogonal rational function $f_{n}\left(z, \tau_{n}\right)$, given by (4.5) has $n$ simple zeros which lie on $\mathbb{T}$.

For our purposes, it is convenient to recall a result about rational Szegö formulas. That are quadrature formulas approximating integrals of the form $\int_{-\pi}^{\pi} f\left(e^{i \theta}\right) h(\theta) d \theta$. Their nodes are the zeros of the para-orthogonal functions $f_{n}$ for the weight $h$. This choice, together with an appropriate choice for the weights, ensures a maximal domain of validity [2, p. 108]. We denote them by $S_{n}(f)=\sum_{j=1}^{n} \lambda_{j, n} f\left(x_{j, n}\right)$ in order to distinguish them from the quadrature formulas $I_{n}$ in (2.1) which approximate integrals (1.2). We have

Theorem 4.2 [2, Theorem 6] Consider a quadrature formula $S_{n}(f)=\sum_{j=1}^{n} \lambda_{j, n} f\left(x_{j, n}\right)$ where $x_{j, n} \neq x_{k, n}$ for $j \neq k$ and $\left|x_{j, n}\right|=1$. Then $S_{n}$ is exact in $\mathcal{R}_{n-1, n-1}$, that is $S_{n}(f)=\int_{-\pi}^{\pi} f\left(e^{i \theta}\right) h(\theta) d \theta$ for all $f \in \mathcal{R}_{n-1, n-1}$ if and only if (i) and (ii) hold:

(i) $I_{n}(f)$ is exact in $\mathcal{R}_{p, q}$ for any pair $(p, q)$ of nonnegative integers satisfying $p+q=n-1$.

(ii) There exist $\tau_{n} \in \mathbb{T}$ such that the nodes $x_{j, n}$ are the zeros of the para-orthogonal rational functions $f_{n}$ from (4.5). 
Since $S_{n}(f)$ is exact for any $f \in \mathcal{R}_{p, q}$, where $p+q=n-1$, we have for the weights

$$
\lambda_{j, n}=\int_{-\pi}^{\pi} L_{j, n}^{p}(x) h(\theta) d \theta, \quad x=e^{i \theta}, \quad j=1,2, \ldots, n
$$

where the $L_{j, n}^{p}$ are given by (2.23). Furthermore, para-orthogonality allows us to write

$$
\lambda_{j, n}=\int_{-\pi}^{\pi}\left|L_{j, n}^{p}(x)\right|^{2} h(\theta) d \theta, \quad x=e^{i \theta} .
$$

Thus, by (2.23) and (4.6) one has

$$
\lambda_{j, n}=\left|\frac{\omega_{p}\left(x_{j, n}\right) \pi_{q}\left(x_{j, n}\right)}{P_{n}^{\prime}\left(x_{j, n}\right)}\right|^{2} \int_{-\pi}^{\pi}\left|\frac{P_{n}(x)}{\left(x-x_{j, n}\right) \omega_{p}(x) \pi_{q}(x)}\right|^{2} h(\theta) d \theta .
$$

If we choose the table $X=\left\{x_{j, n}\right\}$ such that $\left\{x_{j, n}\right\}_{j=1}^{n}$ are the zeros of the $f_{n}\left(z ; \tau_{n}\right)$ given by (4.5), we can prove

Lemma 4.3 Assume that $h$ and $w$ satisfy conditions (4.1)-(4.3) and that $\alpha$ is compactly included in $\mathbb{D}$. Take $X=\left\{x_{j, n}\right\}$ such that $\left\{x_{j, n}\right\}_{j=1}^{n}$ are the zeros of the para-orthogonal rational functions (4.5). Then there exists an absolute constant $C_{2}$ such that

$$
\sum_{j=1}^{n}\left|A_{j, n}\right| \leq C_{2} \sqrt{n}
$$

where the $A_{j, n}$ are the coefficients in the n-point quadrature formula relative to the pair $(\alpha, X)$.

Proof. Multiply and divide the integrand in (2.13) by $\sqrt{h(\theta)}$, and use the Cauchy-Schwarz inequality, (4.3), and (4.7) to get

$$
\left|A_{j, n}\right| \leq\left|\frac{\omega_{p}\left(x_{j, n}\right) \pi_{q}\left(x_{j, n}\right)}{P_{n}^{\prime}\left(x_{j, n}\right)}\right|\left[\int_{-\pi}^{\pi}\left|\frac{P_{n}(x)}{\left(x-x_{j, n}\right) \omega_{p}(x) \pi_{q}(x)}\right|^{2} h(\theta)\right]^{1 / 2}\left[\int_{-\pi}^{\pi} \frac{|w(\theta)|^{2} d \theta}{h(\theta)}\right]^{1 / 2}=\sqrt{C_{1} \lambda_{j, n}}
$$

Since $1 \in \mathcal{R}_{n-1, n-1}$ for each $n=1,2, \ldots$, we get $\sum_{1}^{n} \lambda_{j, n}=\int_{-\pi}^{\pi} h(\theta) d \theta=C_{3}$. Therefore, using (3.6) and the Cauchy-Schwarz inequality gives

$$
\sum_{j=1}^{n}\left|A_{j, n}\right| \leq \sqrt{C_{1}} \sum_{j=1}^{n} \sqrt{\lambda_{j, n}} \leq \sqrt{C_{1} n}\left[\sum_{j=1}^{n} \lambda_{j, n}\right]^{1 / 2}=\sqrt{C_{1} C_{3} n}
$$

which proves (4.8).

A similar result can be proved when the $\left\{x_{j, n}\right\}$ are the zeros of para-orthogonal polynomials with respect to certain rational modifications of a positive weight function. Indeed, set $T_{n-1}(x)=\pi_{q}(x) \omega_{p}(x) \in \Pi_{n-1}($ recall $p+q=n-1)$ and write

$$
h_{n}(\theta)=\frac{h(\theta)}{\left|T_{n-1}\left(e^{i \theta}\right)\right|^{2}} \geq 0, \quad \theta \in[-\pi, \pi] .
$$

Then we have $\int_{-\pi}^{\pi} h_{n}(\theta) d \theta<\infty$. Let $\phi_{n}$ be the orthonormal polynomial of degree $n$ with respect to the measure $h_{n}(\theta) d \theta$ (varying with $n$ ) and let $P_{n}$ be the associated para-orthogonal 
polynomial, i.e., $P_{n}(z)=\phi_{n}(z)+\tau_{n} \phi_{n}^{*}(z), \tau_{n} \in \mathbb{T}$. As mentioned before, $P_{n}$ has exactly $n$ distinct zeros $\left\{x_{j, n}\right\}$ on $\mathbb{T}$. From the Szegö quadrature formula $[15, \S 7]$ we know that there exist $n$ positive numbers $\gamma_{j, n}$ such that for all $R \in \Lambda_{-(n-1), n-1}$

$$
\int_{-\pi}^{\pi} R(x) h_{n}(\theta) d \theta=\sum_{j=1}^{n} \gamma_{j, n} R\left(x_{j, n}\right), \quad x=e^{i \theta}
$$

and

$$
\gamma_{j, n}=\frac{1}{\left|P_{n}^{\prime}\left(x_{j, n}\right)\right|^{2}} \int_{-\pi}^{\pi}\left|\frac{P_{n}(x)}{x-x_{j, n}}\right|^{2} h_{n}(\theta) d \theta=\frac{1}{\left|P_{n}^{\prime}\left(x_{j, n}\right)\right|^{2}} \int_{-\pi}^{\pi} \frac{\left|P_{n}(x)\right|^{2}}{\left|T_{n-1}(x)\right|^{2}} \frac{h(\theta) d \theta}{\left|x-x_{j, n}\right|^{2}} .
$$

Now, multiplying and dividing each term on the right-hand side of (4.11) by $\left|T_{n-1}\left(x_{j, n}\right)\right|^{2}$, we obtain

$$
\int_{-\pi}^{\pi} \frac{R(x) h(\theta)}{\left|T_{n-1}(x)\right|^{2}} d \theta=\sum_{j=1}^{n} \tilde{\gamma}_{j, n} \frac{R\left(x_{j, n}\right)}{\left|T_{n-1}\left(x_{j, n}\right)\right|^{2}}, \quad \forall R \in \Lambda_{-(n-1), n-1}
$$

with

$$
\tilde{\gamma}_{j, n}=\left|\frac{T_{n-1}\left(x_{j, n}\right)}{P_{n}^{\prime}\left(x_{j, n}\right)}\right|^{2} \int_{-\pi}^{\pi}\left|\frac{P_{n}(x)}{T_{n-1}(x)}\right|^{2} \frac{h(\theta)}{\left|x-x_{j, n}\right|^{2}} d \theta, \quad x=e^{i \theta} .
$$

Proceeding as in Lemma 4.3, we see from (4.13) that there exists a positive constant $C_{1}^{\prime}$ such that

$$
\left|A_{j, n}\right| \leq \sqrt{C_{1}^{\prime} \tilde{\gamma}_{j, n}}
$$

On the other hand,

$$
\left|T_{n-1}(x)\right|^{2}=\left|\omega_{p}(x) \pi_{q}(x)\right|^{2}=\omega_{p}(x) \pi_{q}(x) \overline{\omega_{p}(x) \pi_{q}(x)}=\frac{\omega_{p}(x) \pi_{q}(x) \pi_{p}(x) \omega_{q}(x)}{x^{p+q}}
$$

and since $p+q=n-1$, we have $\left|T_{n-1}(x)\right|^{2} \in \Lambda_{-(n-1), n-1}$. If we take $R(x)=\left|T_{n-1}(x)\right|^{2}$ in (4.12), it follows that

$$
\int_{-\pi}^{\pi} h(\theta) d \theta=\sum_{j=1}^{n} \tilde{\gamma}_{j, n}
$$

From (4.14)-(4.15), a result similar to Lemma 4.3 can be proved.

Lemma 4.4 Assume that $h$ and $w$ are as in Lemma 4.3 and take $X=\left\{x_{j, n}\right\}$ such that $\left\{x_{j, n}\right\}_{1}^{n}$ are the zeros of the para-orthogonal polynomials of degree $n$ with respect to the varying measure $h(\theta)\left|\omega_{p}\left(e^{i \theta}\right) \pi_{q}\left(e^{i \theta}\right)\right|^{-2}, p+q=n-1$, then there exists an absolute constant $C_{2}^{\prime}$ such that

$$
\sum_{j=1}^{n}\left|A_{j, n}\right| \leq C_{2}^{\prime} \sqrt{n}
$$

Remark 5. From the point of view of the convergence of quadrature formulas for continuous functions, the meaning of Lemma's 4.3 and 4.4 will become clear later. It follows that taking as nodes the zeros of the para-orthogonal rational functions with respect to a given positive measure $h(\theta) d \theta$ is equivalent to taking nodes as zeros of para-orthogonal polynomials with respect to an appropriate rational modification of this measure. We illustrate this with an example. 
Example. Let us consider the Lebesgue measure $h(\theta)=1 /[2 \pi], \theta \in[-\pi, \pi]$. The orthonormal rational functions are then given by (see $[7$, formula $(1.2)],[5$, p. 165])

$$
\phi_{n}(z)=\sqrt{1-\left|\alpha_{n}\right|^{2}} \frac{z B_{n}(z)}{z-\alpha_{n}} .
$$

Thus, the zeros $\left\{x_{j, n}\right\}$ of $f_{n}(z)=\phi_{n}(z)+\tau_{n} \phi_{n}^{*}(z), \tau_{n} \in \mathbb{T}$ are the roots of the equation

$$
0=P_{n}(z)=z \omega_{n-1}(z)+\tau_{n} \pi_{n-1}(z) \text {. }
$$

On the other hand, for a given polynomial $h(z)$ of degree $k$ of the form $h(z)=h_{1}(z) h_{2}(z)=$ $\prod_{1}^{k_{1}}\left(z-a_{j}\right) \prod_{1}^{k_{2}}\left(1-z b_{j}\right)$ such that $\left|a_{j}\right|<1,\left|b_{j}\right|<1$, and $k_{1}+k_{2}=k$, the $n$th Szegö polynomial with respect to the measure

$$
\frac{d \theta}{2 \pi\left|h\left(e^{i \theta}\right)\right|^{2}}
$$

can be expressed as

$$
\rho_{n}(z)=z^{n-k} h_{1}(z) h_{2}^{*}(z), \quad n \geq k .
$$

The study of rational modifications of a measure has been introduced by Szegö in the case of the unit circle $[21, \S 11.2]$. For further details, see $[13, \S 4]$ for the case of the Lebesgue measure on $\mathbb{T}$ and also [9] where formulas are given for the sequence of monic orthogonal polynomials associated with a rational modification of a finite positive measure supported on Jordan curves. Let us now take $h_{1}(z)=\omega_{p}(z)$ and $h_{2}(z)=\pi_{q}(z), p+q=n-1$, then from (4.19)

$$
\rho_{n}(z)=z \omega_{p}(z) \omega_{q}(z) .
$$

Hence, for $\tau_{n} \in \mathbb{T}$, the zeros of the $n$th corresponding para-orthogonal polynomial are the roots of the equation

$$
0=P_{n}(z)=z \omega_{p}(z) \omega_{q}(z)+\tau_{n} \pi_{p}(z) \pi_{q}(z) .
$$

The nodes of Lemma 4.3 are given by (4.18), while the nodes of Lemma 4.4 are given by (4.21).

Assume that $\{p(n)\}$ and $\{q(n)\}$ are two sequences of nonnegative integers such that $p(n)+q(n)=n-1$ and $\lim _{n \rightarrow \infty} p(n) / n=r<1$. Define for a continuous function $f$ on $\mathbb{T}$

$$
\gamma_{n-1}(f)=\inf _{R \in \mathcal{R}_{n-1, n-1}}\|f-R\|_{\mathbb{T}}
$$

By Theorem 2.1, one has that $\lim _{n \rightarrow \infty} \gamma_{n-1}(f)=0$. However, it can be seen from Lemma 4.3 (or 4.4) that this is not sufficient to assure the convergence of the sequence $\left\{I_{n}(f)\right\}$. So, we need to give estimates of $\gamma_{n-1}(f)$ in terms of $n$ when $f$ is a continuous function on $\mathbb{T}$. Such estimates are known when $f$ is approximated by Laurentpolynomials. More precisely, it is proved in [4, Theorem 5] by using Jackson's theorem III (see [6, Ch. 4, § 6, p. 144]) that if $f$ is a continuous function on $\mathbb{T}$ then there exists some $R_{n-1} \in \Lambda_{-(n-1), n-1}$ such that

$$
\max _{x \in \mathbb{T}}\left|f(x)-R_{n-1}(x)\right| \leq 2 \omega(f, \pi / n),
$$

where $\omega(f, \delta)=\sup \left\{\left|f\left(e^{i s}\right)-f\left(e^{i t}\right)\right|:|t-s|<\delta ;-\pi \leq s, t \leq \pi\right\}$ denotes the modulus of continuity of $f$ on $\mathbb{T}$. If $\alpha_{0} \neq 0$ is a fixed point in $\mathbb{D}$, then a bilinear transformation gives

$$
\gamma_{n-1}(f) \leq K \omega(f, \pi / n)
$$


where $K$ is a constant and

$$
\gamma_{n-1}(f)=\inf _{P \in \Pi_{2 n-2}}\left\|f(z)-\frac{P(z)}{\left(z-\alpha_{0}\right)^{n-1}\left(1-\bar{\alpha}_{0} z\right)^{n-1}}\right\|_{\mathbb{T}} .
$$

Thus, it seems natural to wonder if the same type of estimate for $\gamma_{n-1}(f)$ holds when $f$ is approximated by functions from $\mathcal{R}_{n-1, n-1}$ (recall that $\mathcal{R}_{n-1, n-1}$ depends on the sequence $\left.\alpha=\left\{\alpha_{k}\right\} \subset \mathbb{D}\right)$. In this respect, a Jackson-type theorem was proved in [11, Thm. 4] for a function continuous on $[-1,1]$ that is approximated by rational functions with prescribed poles outside $[-1,1]$. We shall prove a similar result. First some auxiliary results are required. Let

$$
L_{n}(z)=\frac{P_{2 n}}{z^{n}} \in \Lambda_{-n, n}, \quad P_{2 n} \in \Pi_{2 n}
$$

be a Laurent polynomial and $T_{n}(\theta)=L_{n}\left(e^{i \theta}\right)$ a trigonometric polynomial of degree $n$. Then it is clear that $\left|T_{n}(\theta)\right|=\left|L_{n}(z)\right|=\left|P_{2 n}(z)\right|$ for $z=e^{i \theta} \in \mathbb{T}$. We also consider the trigonometric rational function $R_{n}(\theta)=T_{n}(\theta)\left|\omega_{n}\left(e^{i \theta}\right)\right|^{-2}$. If $\left\{\theta_{j}\right\}_{j=1}^{2 n+1} \subset[-\pi, \pi]$ with $\theta_{j} \neq \theta_{k}$ for $j \neq k$ and $f$ is a periodic function on $[-\pi, \pi]$, then $R_{n}(\theta)$ is uniquely defined by the interpolation conditions

$$
R_{n}\left(\theta_{j}\right)=f\left(\theta_{j}\right), \quad j=1,2, \ldots, 2 n+1,
$$

because the system $\{1, \sin \theta, \ldots, \sin n \theta, \cos \theta, \ldots, \cos n \theta\}$ satisfies the Haar condition. We have

$$
R_{n}(\theta)=\tilde{R}_{n}\left(e^{i \theta}\right)=\frac{P_{2 n}\left(e^{i \theta}\right)}{e^{i n \theta}\left|\omega_{n}\left(e^{i \theta}\right)\right|^{2}}=\frac{P_{2 n}\left(e^{i \theta}\right)}{\omega_{n}\left(e^{i \theta}\right) \pi_{n}\left(e^{i \theta}\right)} \in \mathcal{R}_{n, n} .
$$

Setting $x_{j}=e^{i \theta_{j}}, j=1,2, \ldots, 2 n+1$, we have $\tilde{R}_{n}\left(x_{j}\right)=g\left(x_{j}\right)$, where $g\left(e^{i \theta}\right)=f(\theta)$. Let us assume that $g$ is defined on a certain neighborhood $G$ including $\mathbb{T}$ and let $\Gamma$ be the boundary of $G$. Then, from Hermite's interpolation formula (see Walsh [24, Thm. 2, Ch. 8]), one has

$$
\begin{aligned}
R_{n}(\theta)-f(\theta) & =\tilde{R}_{n}\left(e^{i \theta}\right)-g\left(e^{i \theta}\right)=\tilde{R}_{n}(z)-g(z), \quad z=e^{i \theta} \\
& =\int_{\Gamma} \frac{\left(z-x_{1}\right) \cdots\left(z-x_{2 n+1}\right) \omega_{n}(t) \pi_{n}(t)}{\left(t-x_{1}\right) \cdots\left(t-x_{2 n+1}\right) \omega_{n}(z) \pi_{n}(z)} \frac{g(t)}{z-t} d t \\
& =\int_{\Gamma} \frac{\left[\chi_{2 n+1}(z)\right] /\left[\omega_{n}(z) \pi_{n}(z)\right]}{\left[\chi_{2 n+1}(t)\right] /\left[\omega_{n}(t) \pi_{n}(t)\right]} \frac{g(t)}{z-t} d t,
\end{aligned}
$$

where $\chi_{k}(z)=\Pi_{j=1}^{k}\left(z-x_{j}\right)$.

Next we need to extend the Bernstein-Walsh formula (see Walsh [24, p. 77]) to trigonometric polynomials. We recall that, for ordinary polynomials, this formula says that if $f$ is a continuous function on $[a, b]$ and $Q_{m}^{*}$ is the best (in uniform norm) polynomial approximation of degree $m$, then for $m$ sufficiently large

$$
\left|Q_{m}^{*}(z)\right| \leq 2\|f\|_{\infty} \exp \left\{m g_{[a, b]}(z, \infty)\right\},
$$

where $g_{[a, b]}(z, \infty)$ is Green's function for the region $\hat{\mathbb{C}}-[a, b]$ with singularity at infinity. The trigonometric generalization of this result is

Lemma 4.5 Let $f$ be a real $2 \pi$-periodic continuous function and let $T_{m}^{*}(\theta)$ be the trigonometric polynomial of degree $m$ of best approximation in the uniform norm. Then if $L_{m}^{*} \in \Lambda_{-m, m}$ is the Laurent polynomial such that $T_{m}^{*}(\theta)=L_{m}^{*}\left(e^{i \theta}\right)$, for sufficiently large $m$ we have

(i) $\forall z \neq 0, \quad|z| \leq 1: \quad\left|L_{m}^{*}(z)\right| \leq 2\|f\|_{\infty}|z|^{-m}$,

(ii) $\forall z \in \mathbb{C}, \quad|z| \geq 1:\left|L_{m}^{*}(z)\right| \leq 2\|f\|_{\infty}|z|^{m}$. 
Proof. Given $P_{2 m}^{*} \in \Pi_{2 m}$, put $L_{m}^{*}(z)=P_{2 m}^{*}(z) / z^{m}$. Since $P_{2 m}^{*}$ is analytic in $\mathbb{D} \cup \mathbb{T}$, we have that

$$
\left|P_{2 m}^{*}(z)\right| \leq \max _{|z| \leq 1}\left|P_{2 m}^{*}(z)\right|=\max _{z \in \mathbb{T}}\left|P_{2 m}^{*}(z)\right|=\left\|T_{m}^{*}\right\|_{\infty}, \quad z \in \mathbb{D} \cup \mathbb{T} .
$$

Thus, if $z \neq 0$ and $|z| \leq 1$ then

$$
\left|L_{m}^{*}(z)\right|=\left|\frac{P_{2 m}^{*}(z)}{z^{m}}\right| \leq\left\|T_{m}^{*}\right\|_{\infty}|z|^{-m}
$$

For $z \in \mathbb{C}$ and $|z| \geq 1$ we consider the function $z^{-2 m} P_{2 m}^{*}(z)$, analytic in $|z| \geq 1$, so that

$$
\left|\frac{L_{m}^{*}(z)}{z^{m}}\right|=\left|\frac{P_{2 m}^{*}(z)}{z^{2 m}}\right| \leq \max _{|z| \geq 1}\left|\frac{P_{2 m}^{*}(z)}{z^{2 m}}\right|=\max _{z \in \mathbb{T}}\left|P_{2 m}^{*}(z)\right|=\left\|T_{m}^{*}\right\|_{\infty} .
$$

By the Weierstrass theorem, we have $\lim _{m \rightarrow \infty} T_{m}^{*}(\theta)=f(\theta)$ uniformly on $[-\pi, \pi]$. Thus for sufficiently large $m$

$$
\left\|T_{m}^{*}\right\|_{\infty} \leq 2\|f\|_{\infty}
$$

By (4.25)-(4.27) the result now follows.

Now we can prove a rational form of Jackson's theorem III.

Lemma 4.6 Let $\alpha=\left\{\alpha_{k}\right\}$ be compactly included in $\mathbb{D}$ such that (3.4)-(3.5) hold for $r=s=$ $1 / 2$ and let $f$ be a real $2 \pi$-periodic continuous function. Then there exists a trigonometric rational function $R_{n}(\theta)=T_{n}(\theta)\left|\omega\left(e^{i \theta}\right)\right|^{-2}$ with $T_{n}(\theta)$ a trigonometric polynomial such that for sufficiently large $n$ there is a constant $K_{2}$ with

$$
\left\|f-R_{n}\right\|_{\infty} \leq K_{2} \omega\left(f, \frac{\pi}{n+1}\right) .
$$

Proof. Let $T_{m}^{*}$ be the best trigonometric polynomial approximation to $f$ of degree $m$ and set $T_{m}^{*}(\theta)=L_{m}^{*}\left(e^{i \theta}\right), L_{m}^{*} \in \Lambda_{-m, m}$. Let $X=\left\{X_{n}\right\}_{n=1}^{\infty}$ with $X_{n}=\left\{x_{j, n}: j=1, \ldots, n\right\}$ be a triangular table of points contained in $\mathbb{T}$. Set $\chi_{n}(z)=\Pi_{j=1}^{n}\left(z-x_{j, n}\right)$ and, with the notation introduced above, assume that

$$
\nu_{n}^{X} \stackrel{*}{\rightarrow} \tilde{\nu}
$$

where $\nu_{n}^{X}=\nu\left(\chi_{n}\right)$ and $\tilde{\nu}$ is the equilibrium measure on $\mathbb{T}$ in the presence of the exterior field

$$
-V(z)=-\frac{1}{2}\left(V_{\nu^{\alpha}}(z)+V_{\nu^{\alpha}}(1 / z)+V_{\delta_{0}}(z)\right)
$$

here $\delta_{0}$ is the Dirac measure supported at $z=0$. Details about existence and construction of table $X$ can be found in [10, main thm. p. 124]. Let $T_{m}^{*}$ be the trigonometric polynomial of degree $m$ that is the best approximation of $f$ and suppose that $T_{m}^{*}(\theta)=L_{m}^{*}\left(e^{i \theta}\right), L_{m}^{*} \in$ $\Lambda_{-m, m}$. Set $x_{j, n}=e^{i \theta_{j, n}}, j=1, \ldots, n$ and take $R_{n}(\theta)=T_{n}(\theta)\left|\omega_{n}\left(e^{i \theta}\right)\right|^{-2}$ the trigonometric rational function of degree $n$ which interpolates $T_{m}^{*}$ at $\theta=\theta_{j, 2 n+1}, j=1, \ldots, 2 n+1$. By (4.24), for $z=e^{i \theta} \in \mathbb{T}$ we have

$$
R_{n}(\theta)-T_{m}^{*}(\theta)=\int_{\Gamma} \frac{\frac{\chi_{2 n+1}(z)}{\omega_{n}(z) \pi_{n}(z)}}{\frac{\chi_{2 n+1}(t)}{\omega_{n}(t) \pi_{n}(t)}} \frac{L_{m}^{*}(t)}{z-t} d t
$$

Suppose that $\Gamma=\Gamma_{1} \cup \Gamma_{2}$ where $\Gamma_{1}=\{z \in \mathbb{C}:|z|=r<1\}$ and $\Gamma_{2}=\{z \in \mathbb{C}:|z|=R>1\}$. If $G$ denotes the annulus centered at the origin with radii $r$ and $R$, then, since $\alpha$ is compactly 
included in $\mathbb{D}, r$ and $R$ can be chosen sufficiently close to 1 so that $G \cap(\alpha \cup \hat{\alpha})=\emptyset$. Setting $\rho=\log (\max \{R, 1 / r\})$, by the preceding lemma, we obtain

$$
\sup _{x \in \Gamma}\left|L_{m}^{*}(x)\right| \leq 2\|f\|_{\infty} \exp \{m \rho\} .
$$

Replacing $n$ by $2 n+1$ in (3.4) and (3.5) and setting $p(2 n+1)=q(2 n+1)=n$, so that $r=s=1 / 2$, for $z \in(\mathbb{C}-\{0\})-\operatorname{supp}\left(\nu^{\alpha}\right)$ and $1 / z \notin \operatorname{supp}\left(\nu^{\bar{\alpha}}\right)$ one obtains

$$
\lim _{n \rightarrow \infty}\left|\omega_{n}(z) \pi_{n}(z)\right|^{1 /(2 n+1)}=\exp \left\{-\frac{1}{2}\left(V_{\nu^{\alpha}}(z)+V_{\nu^{\bar{\alpha}}}(1 / z)\right)\right\}|z|^{1 / 2}=\exp \{-V(z)\}
$$

and, for $z \neq 0$,

$$
\limsup _{n \rightarrow \infty}\left|\omega_{n}(z) \pi_{n}(z)\right|^{1 /(2 n+1)} \leq \exp \{-V(z)\} .
$$

By (4.29) and (4.30) it follows that

$$
\left|R_{n}(\theta)-T_{m}^{*}(\theta)\right| \leq K_{1} \frac{\sup _{z \in \mathbb{T}}\left|\frac{\chi_{2 n+1}(z)}{\omega_{n}(z) \pi_{n}(z)}\right|}{\inf _{t \in \Gamma}\left|\frac{\chi_{2 n+1}(t)}{\omega_{n}(t) \pi_{n}(t)}\right|} \exp \{m \rho\}\|f\|_{\infty} .
$$

By (4.31), we have

$$
\lim _{n \rightarrow \infty}\left|\frac{\chi_{2 n+1}(t)}{\omega_{n}(t) \pi_{n}(t)}\right|^{1 /(2 n+1)}=\exp \left\{V(t)-V_{\tilde{\nu}}(t)\right\}
$$

uniformly for $t \in \Gamma$, and, by (4.32)

$$
\underset{n \rightarrow \infty}{\limsup }\left|\frac{\chi_{2 n+1}(z)}{\omega_{n}(z) \pi_{n}(z)}\right|^{1 /(2 n+1)} \leq \exp \left\{V(z)-V_{\tilde{\nu}}(z)\right\}
$$

uniformly on $\mathbb{T}$. Given $\epsilon>0$, we obtain

$$
\left|\frac{\chi_{2 n+1}(t)}{\omega_{n}(t) \pi_{n}(t)}\right| \geq \exp \left\{(2 n+1)\left[V(t)-V_{\tilde{\nu}}(t)-\epsilon\right]\right\}
$$

for $n \geq n_{0}$ and $t \in \Gamma$, and,

$$
\left|\frac{\chi_{2 n+1}(z)}{\omega_{n}(z) \pi_{n}(z)}\right| \leq \exp \left\{(2 n+1)\left[V(z)-V_{\tilde{\nu}}(z)+\epsilon\right]\right\}=\exp \{(2 n+1)(K+\epsilon)\}
$$

for $z \in \mathbb{T}$, where $K$ is the constant value that $V(z)-V_{\tilde{\nu}}(z)$ takes on $\mathbb{T}$. From (4.33) and the last two centered formulas we can conclude that, for $n \geq n_{0}$ and $\theta \in[-\pi, \pi]$,

$$
\left|R_{n}(\theta)-T_{m}^{*}(\theta)\right| \leq K_{1}\|f\|_{\infty} \exp \{m \rho\} \exp \left\{(2 n+1)\left[K+2 \epsilon-\inf _{t \in \Gamma}\left(V(t)-V_{\tilde{\nu}}(t)\right)\right]\right\} .
$$

Take $m=m(n)=\lfloor(2 n+1) / l\rfloor$ with $\lfloor\cdot\rfloor$ the integer part and $l$ an appropriately chosen positive constant. Then $m \leq(2 n+1) / l$ and from (4.34) it follows

$$
\left|R_{n}(\theta)-T_{m(n)}^{*}(\theta)\right| \leq K_{1}\|f\|_{\infty} \exp \left\{(2 n+1)\left[\rho / l+K+2 \epsilon-\inf _{t \in \Gamma}\left(V(t)-V_{\tilde{\nu}}(t)\right)\right]\right\} .
$$

On the other hand, it is known that

$$
\inf _{t \in \Gamma}\left(V(t)-V_{\tilde{\nu}}(t)\right)>K .
$$


Therefore, by taking $\epsilon>0$ sufficiently small and $l$ sufficiently large, we can ensure that

$$
\rho / l+K+2 \epsilon-\inf _{t \in \Gamma}\left(V(t)-V_{\tilde{\nu}}(t)\right)=-C
$$

with $C$ a positive constant. Thus we have obtained that for all $\theta \in[-\pi, \pi]$

$$
\left|R_{n}(\theta)-T_{m}^{*}(\theta)\right| \leq K_{1}\|f\|_{\infty} \exp \{-C(2 n+1)\} .
$$

Next we assume without loss of generality that $f$ vanishes at least once at a point $\theta_{0} \in[-\pi, \pi]$ (if not, it suffices to consider $g(\theta)=f(\theta)-f\left(\theta_{0}\right)$ and take into account that $\omega(f, \delta)=\omega(g, \delta)$ ). Under this condition

$$
\|f\|_{\infty} \leq \max _{\theta \in[-\pi, \pi]}\{f(\theta)\}-\min _{\theta \in[-\pi, \pi]}\{f(\theta)\} .
$$

Let $f\left(\theta_{1}\right)$ be the maximum and $f\left(\theta_{2}\right)$ be the minimum. Suppose $\theta_{1}<\theta_{2}$ (the other case is similar); then

$$
\begin{aligned}
\left|f\left(\theta_{1}\right)-f\left(\theta_{2}\right)\right| & =\left|\sum_{i=0}^{2 n+1}\left[f\left(\theta_{1}+\frac{i\left(\theta_{2}-\theta_{1}\right)}{2 n+2}\right)-f\left(\theta_{1}+\frac{(i+1)\left(\theta_{2}-\theta_{1}\right)}{2 n+2}\right)\right]\right| \\
& \leq \sum_{i=0}^{2 n+1}\left|f\left(\theta_{1}+\frac{i\left(\theta_{2}-\theta_{1}\right)}{2 n+2}\right)-f\left(\theta_{1}+\frac{(i+1)\left(\theta_{2}-\theta_{1}\right)}{2 n+2}\right)\right| \\
& \leq(2 n+2) \omega\left(f, \frac{\pi}{n+1}\right) .
\end{aligned}
$$

Therefore

$$
\|f\|_{\infty} \leq(2 n+2) \omega\left(f, \frac{\pi}{n+1}\right) .
$$

From (4.35) and (4.37) we obtain

$$
\left|R_{n}(\theta)-T_{m}^{*}(\theta)\right| \leq K_{1} \omega\left(f, \frac{\pi}{n+1}\right) \exp \{\log (2 n+2)-C(2 n+1)\} .
$$

Now, using Jackson's theorem III [6, p. 144], for $2 n+1 \geq l$ we obtain

$$
\begin{aligned}
\left|f(\theta)-T_{m(n)}^{*}(\theta)\right| & \leq 2 \omega\left(f, \frac{\pi}{m(n)+1}\right) \leq 2 \omega\left(f, \frac{\pi}{\left[\frac{2 n+1}{l}\right]}\right) \\
& \leq 2 \omega\left(f, \frac{2 l \pi}{2 n+1}\right) \leq 2 \omega\left(f, \frac{2 l \pi}{n+1}\right) \\
& \leq 2(2 l+1) \omega\left(f, \frac{\pi}{n+1}\right),
\end{aligned}
$$

where we have used that $\left\lfloor\frac{n}{l}\right\rfloor \geq \frac{n}{2 l}$ if $n \geq l$, that $\omega\left(f, \delta_{1}\right) \leq \omega\left(f, \delta_{2}\right)$ if $\delta_{1} \leq \delta_{2}$ and that $\omega(f, \lambda \delta) \leq(1+\lambda) \omega(f, \delta)$. A combination of (4.39) and (4.38) then gives

$$
\begin{aligned}
\left|f(\theta)-R_{n}(\theta)\right| & \leq\left|f(\theta)-T_{m(n)}^{*}(\theta)\right|+\left|T_{m(n)}^{*}(\theta)-R_{n}(\theta)\right| \\
& \leq\left[2(2 l+1)+K_{1} \exp \{\log (2 n+2)-C(2 n+1)\}\right] \omega\left(f, \frac{\pi}{n+1}\right) .
\end{aligned}
$$

Hence, noting that $\lim _{n \rightarrow \infty} \exp [\log (2 n+2)-C(2 n+1)]=0$, the lemma follows.

Finally we are ready to prove

Theorem 4.7 Let $\alpha=\left\{\alpha_{k}\right\}$ be compacly included in $\mathbb{D}$ such that (3.4)-(3.5) hold for $r=$ $s=1 / 2$ and let $f$ be a function continuous on $\mathbb{T}$. Then there exists a constant $C_{4}$ such that

$$
\gamma_{n}(f) \leq C_{4} \omega\left(f, \frac{\pi}{n+1}\right),
$$

where $\gamma_{n}(f)$ is as in (4.22) with $n$ sufficiently large. 
Proof. Let us now consider a continuous function $f$ on $\mathbb{T}$; then we can set

$$
f\left(e^{i \theta}\right)=f_{1}(\theta)+i f_{2}(\theta), \quad \theta \in[-\pi, \pi]
$$

where $f_{1}(\theta)=\Re\left(f\left(e^{i \theta}\right)\right)$ and $f_{2}(\theta)=\Im\left(f\left(e^{i \theta}\right)\right)$ are $2 \pi$-periodic functions on $[-\pi, \pi]$. Let $R_{n, j}(\theta), j=1,2$ be the trigonometric rational functions appearing in (4.28) and corresponding to $f_{j}(\theta), j=1,2$, that is,

$$
\left\|f_{j}-R_{n, j}\right\|_{\infty} \leq K_{2, j} \omega\left(f_{j}, \frac{\pi}{n+1}\right), \quad j=1,2 .
$$

Write $R_{n}(z)=R_{n, 1}(\theta)+i R_{n, 2}(\theta), z=e^{i \theta}$; then clearly $R_{n} \in \mathcal{R}_{n, n}$. Furthermore, since $\left|f_{j}(s)-f_{j}(t)\right| \leq\left|f\left(e^{i s}\right)-f\left(e^{i t}\right)\right|, j=1,2$, it follows that $\omega\left(f_{j}, \delta\right) \leq \omega(f, \delta), j=1,2$. Thus, by (4.28)-(4.41) it follows that

$$
\gamma_{n}(f) \leq \max _{z \in \mathbb{T}}\left|f(z)-R_{n}(z)\right| \leq\left\|f_{1}-R_{n, 1}\right\|_{\infty}+\left\|f_{2}-R_{n, 2}\right\|_{\infty} \leq\left(K_{2,1}+K_{2,2}\right) \omega\left(f, \frac{\pi}{n+1}\right) .
$$

This concludes the proof.

It follows that if $f$ is a continuous function on $\mathbb{T}$ with $\omega(f, \delta)=O\left(\delta^{p}\right)$ and $p>1 / 2$, then, by Theorem 4.7 and Lemma 4.3 or 4.4, there exist constants $A$ and $B$ independent of $n$ such that for sufficiently large $n$ one gets

$$
\left|I_{n}(f)-I_{\mu}(f)\right| \leq \frac{A+B \sqrt{n}}{n^{p}} .
$$

This means that the following theorem is proved.

Theorem 4.8 Let $f$ be a continuous function on $\mathbb{T}$ with modulus of continuity $\omega(f, \delta)=$ $O\left(\delta^{p}\right)$ for some $p>1 / 2$. Let $X=\left\{x_{j, n}\right\}$ be the triangular array of nodes as described in Lemma 4.3 or as in 4.4. Then the sequence of quadrature formulas with nodes $\left\{x_{j, n}\right\}$ converges to $I_{\mu}(f)$.

Remark 6. Note that Theorem 4.8 holds for any function $f$ satisfying a Lipschitz condition of the form

$$
\left|f\left(z_{1}\right)-f\left(z_{2}\right)\right| \leq \lambda\left|z_{1}-z_{2}\right|^{p}, \quad p>1 / 2 ; \quad z_{1}, z_{2} \in \mathbb{T} .
$$

\section{References}

[1] N.I. Achieser. Theory of approximation. Frederick Ungar Publ. Co., New York, 1956.

[2] A. Bultheel, P. González-Vera, E. Hendriksen, and O. Njåstad. Orthogonal rational functions and quadrature on the unit circle. Numer. Algorithms, 3:105-116, 1992.

[3] A. Bultheel, P. González-Vera, E. Hendriksen, and O. Njåstad. Orthogonality and boundary interpolation. In A.M. Cuyt, editor, Nonlinear Numerical Methods and Rational Approximation II, pages 37-48. Kluwer, 1994.

[4] A. Bultheel, P. González-Vera, E. Hendriksen, and O. Njåstad. Quadrature formulas on the unit circle and two-point Padé approximation. In A.M. Cuyt, editor, Nonlinear Numerical Methods and Rational Approximation II, pages 303-318. Kluwer, 1994. 
[5] A. Bultheel, P. González-Vera, E. Hendriksen, and O. Njåstad. Quadrature formulas on the unit circle based on rational functions. J. Comput. Appl. Math., 50:159-170, 1994.

[6] E.W. Cheney. Introduction to approximation theory. McGraw Hill, 1966.

[7] M.M. Djrbashian. A survey on the theory of orthogonal systems and some open problems. In P. Nevai, editor, Orthogonal polynomials: Theory and practice, volume 294 of Series C: Mathematical and Physical Sciences, pages 135-146, Boston, 1990. NATOASI, Kluwer Academic Publishers.

[8] W. Gautschi. Gauss-type quadrature rules for rational functions. In H. Brass and G. Hammerlin, editors, Numerical integration IV, volume 112 of Internat. Ser. of Numer. Math., pages 111-130, 1993.

[9] E. Godoy and F. Marcellan. Orthogonal polynomials and rational modification of measures. Canad. J. Math., 45:930-943, 1993.

[10] A.A. Gonchar and E.A. Rakhmanov. Equilibrium measure and the distribution of zeros of extremal polynomials. Math. USSR-Sb., 53:119-130, 1986.

[11] P. González-Vera, M. Jiménez-Páiz, G. López-Lagomasino, and R. Orive. On the convergence of quadrature formulas connected with multipoint Padé-type approximants. $J$. Math. Anal. Appl., 1996. To appear.

[12] P. González-Vera, G. López-Lagomasino, R. Orive, and J.C. Santos-Leon. On the convergence of quadrature formulas for complex weight functions. J. Math. Anal. Appl., 189:514-532, 1995.

[13] P. González-Vera, O. Njåstad, and J.C. Santos-Leon. Some numerical results about quadrature formulas on the unit circle. Adv. Comput. Math., 1995. In press.

[14] J. Illan and G. López-Lagomasino. A note on generalized quadrature formulas of GaussJacobi type. In Constructive Theory of Functions '84, Sofia, pages 513-518, 1984.

[15] W.B. Jones, O. Njåstad, and W.J. Thron. Moment theory, orthogonal polynomials, quadrature and continued fractions associated with the unit circle. Bull. London Math. Soc., 21:113-152, 1989.

[16] A. Máté, P. Nevai, and V. Totik. Strong and weak convergence of orthogonal polynomials. Amer. J. Math., 109:239-281, 1987.

[17] J. Nuttal and C.J. Wherry. Gauss integration for complex weight functions. J. Inst. Math. Appl., 21:165-170, 1987.

[18] E.A. Rakhmanov. On the asymptotics of the ratio of orthogonal polynomials. Math. Sb., 32:199-213, 1977.

[19] E.A. Rakhmanov. On the asymptotics of the ratio of orthogonal polynomials II. Math. Sb., 46:105-117, 1983.

[20] H. Stahl and V. Totik. General orthogonal polynomials. Encyclopedia of Mathematics and its Applications. Cambridge University Press, 1992. 
[21] G. Szegö. Orthogonal polynomials, volume 33 of Amer. Math. Soc. Colloq. Publ. Amer. Math. Soc., Providence, Rhode Island, 3rd edition, 1967. First edition 1939.

[22] W. Van Assche and I. Vanherwegen. Quadrature formulas based on rational interpolation. Math. Comp., 16:765-783, 1993.

[23] H. Wallin. Potential theory and approximation of analytic functions by rational interpolation. In I. Laine, O. Letho, and T. Sorvali, editors, Complex analysis, Joensuu 1978, volume 747 of Lecture Notes in Math., pages 434-450. Springer Verlag, 1979.

[24] J. L. Walsh. Interpolation and approximation, volume 20 of Amer. Math. Soc. Colloq. Publ. Amer. Math. Soc., Providence, Rhode Island, 3rd edition, 1960. First edition 1935 . 